\title{
GENERATING FUNCTIONS FOR PLANE OR AXISYMMETRIC ISOCHORIC DEFORMATIONS*
}

BY

\author{
F. J. ROONEY AND M. M. CARROLL \\ University of California, Berkeley
}

\begin{abstract}
Generating functions for canonical transformations of single degree of freedom Hamiltonian systems provide displacement potentials for isochoric plane or axisymmetric finite deformations.
\end{abstract}

Introduction. Velocity fields for plane isochoric motions admit spatial representations in terms of stream functions. Thus, if

$$
\dot{x}=u(x, y, t), \quad \dot{y}=v(x, y, t), \quad \dot{z}=0
$$

and

$$
\partial u / \partial x+\partial v / \partial y=0
$$

then

$$
\dot{x}=\frac{\partial \psi}{\partial y}, \quad \dot{y}=-\frac{\partial \psi}{\partial x} ; \quad \psi=\hat{\psi}(x, y, t) .
$$

Here $x, y, z$ are rectangular Cartesian coordinates of a typical particle at time $t$ and the superposed dot denotes the material time derivative. If $X, Y, Z$ denote the coordinates of the particle in a reference configuration (at time $t=0$, say), then the material description of the motion has the form

$$
x=\hat{x}(X, Y, t), \quad y=\hat{y}(X, Y, t), \quad z=Z
$$

and the condition that the motion be isochoric is

$$
\frac{\partial x}{\partial X} \frac{\partial y}{\partial Y}-\frac{\partial x}{\partial Y} \frac{\partial y}{\partial X}=1 .
$$

In view of the stream function representation (3) of the velocity field, it is of interest to examine whether or not the condition (5) implies a representation of the motion (4) in terms of a single function.

Velocity fields for axisymmetric isochoric motions also admit spatial representations similar to Eq. (3). Thus, if

$$
\dot{r}=u(r, z, t), \quad \dot{\theta}=0, \quad \dot{z}=w(r, z, t)
$$

\footnotetext{
${ }^{*}$ Received June 27, 1983.
} 
and

$$
\partial u / \partial r+u / r+\partial w / \partial z=0
$$

then

$$
\dot{r}=\frac{1}{r} \frac{\partial \psi}{\partial z}, \quad \dot{z}=-\frac{1}{r} \frac{\partial \psi}{\partial r} ; \quad \psi=\tilde{\psi}(r, z, t) .
$$

If $R, \Theta, Z$ denote cylindrical coordinates of the particle in the reference configuration, then the material description of the motion has the form

$$
r=\hat{r}(R, Z, t), \quad \theta=\Theta, \quad z=\hat{z}(R, Z, t)
$$

and the condition that the motion be isochoric is

$$
\frac{r}{R}\left(\frac{\partial r}{\partial R} \frac{\partial z}{\partial Z}-\frac{\partial r}{\partial Z} \frac{\partial z}{\partial R}\right)=1 .
$$

Again, we seek to represent the motion (9) in terms of a single function.

Generating functions for canonical transformations. Hamilton's canonical equations are

$$
\dot{q}=\frac{\partial h}{\partial p}, \quad \dot{p}=-\frac{\partial h}{\partial q} ; \quad h=\hat{h}(p, q, t),
$$

where $q$ and $p$ denote generalized coordinates and momenta and $h$ is the Hamiltonian. In general, $p$ and $q$ are vectors, but for present purposes it suffices to consider a single degree of freedom system, so that $p$ and $q$ are scalars. A transformation

$$
Q=\hat{Q}(p, q, t), \quad P=\hat{P}(p, q, t)
$$

to a new generalized coordinate and momentum is a canonical transformation if there is a function $\hat{H}$ such that

$$
\dot{Q}=\frac{\partial H}{\partial P}, \quad \dot{P}=-\frac{\partial H}{\partial Q} ; \quad \hat{H}(P, Q, t) .
$$

Such transformations are obtained from generating functions $F$, which may be written in any one of four forms (see, for example, Goldstein [1])

$$
F_{1}(q, Q, t), \quad F_{2}(q, P, t), \quad F_{3}(p, Q, t), \quad F_{4}(p, P, t) .
$$

For each form we have

$$
\begin{array}{ll}
p=\partial F_{1} / \partial q, & P=-\partial F_{1} / \partial Q, \\
p=\partial F_{2} / \partial q, & Q=\partial F_{2} / \partial P, \\
q=-\partial F_{3} / \partial p, & p=-\partial F_{3} / \partial Q, \\
q=-\partial F_{4} / \partial p, & Q=\partial F_{4} / \partial P,
\end{array}
$$

with

$$
H_{i}=h+\partial F_{i} / \partial t \quad(i=1,2,3,4)
$$

Generating functions for isochoric plane motions. Equations (3), which describe the stream function representation of isochoric plane velocity fields, are the same as Hamilton's canonical equations (11), with $x$ and $y$ as generalized coordinate and momentum, 
respectively, and $\psi$ as Hamiltonian. With this identification, a plane isochoric motion defines a transformation of the form (12) and, since the stream function representation holds at each instant of time, this is a canonical transformation. We can therefore use any one of the four generating functions (14) to provide a representation of the motion (4) in terms of a single function. For example, we may write

$$
y=\partial F / \partial x, \quad Y=-\partial F / \partial X ; \quad F=F_{1}(x, X, t)
$$

or

$$
y=\partial F / \partial x, \quad X=\partial F / \partial Y ; \quad F=F_{2}(x, Y, t) .
$$

Equations (20) and (21) do not give the motion explicitly in the form of Eqs. (4). A particular choice of generating function $F_{1}$, for example, leads to equations

$$
y=\tilde{y}(x, X, t), \quad Y=\tilde{Y}(x, X, t)
$$

and the explicit form (4) requires inversion of the second of these equations, i.e.,

$$
x=\tilde{Y}^{-1}(Y, X, t)=\hat{x}(X, Y, t)
$$

and

$$
y=\tilde{y}\left(\tilde{Y}^{-1}(Y, X, t), X, t\right)=\hat{y}(X, Y, t) .
$$

In order to calculate the deformation gradients from equations (21), we observe that if

$$
\phi=g(x, Y, t)=G(X, Y, t),
$$

then

$$
\frac{\partial G}{\partial X}=\frac{\partial g}{\partial x} \frac{\partial \hat{x}}{\partial X}, \quad \frac{\partial G}{\partial Y}=\frac{\partial g}{\partial x} \frac{\partial \hat{x}}{\partial Y}+\frac{\partial g}{\partial Y} .
$$

Equations (21), (23) and (24) thus lead to

$$
\frac{\partial \hat{y}}{\partial X}=\frac{\partial^{2} F}{\partial x^{2}} \frac{\partial \hat{x}}{\partial X}, \quad \frac{\partial \hat{y}}{\partial Y}=\frac{\partial^{2} F}{\partial x^{2}} \frac{\partial \hat{x}}{\partial Y}+\frac{\partial^{2} F}{\partial x \delta Y}
$$

and

$$
1=\frac{\partial^{2} F}{\partial x \partial Y} \frac{\partial \hat{x}}{\partial X}, \quad 0=\frac{\partial^{2} F}{\partial x \partial Y} \frac{\partial \hat{x}}{\partial Y}+\frac{\partial^{2} F}{\partial Y^{2}}
$$

so that

$$
\begin{aligned}
& \left(\begin{array}{ll}
\frac{\partial \hat{x}}{\partial X} & \frac{\partial \hat{x}}{\partial Y} \\
\frac{\partial \hat{y}}{\partial X} & \frac{\partial \hat{y}}{\partial Y}
\end{array}\right) \\
& \quad=\left(\begin{array}{lc}
\frac{1}{\partial^{2} F / \partial x \partial Y}
\end{array}\right)\left(\begin{array}{cc}
1 & -\frac{\partial^{2} F}{\partial Y^{2}} \\
\frac{\partial^{2} F}{\partial x^{2}} & \left(\frac{\partial^{2} F}{\partial x \partial Y}\right)^{2}-\frac{\partial^{2} F}{\partial x^{2}} \frac{\partial^{2} F}{\partial Y^{2}}
\end{array}\right)
\end{aligned}
$$


The isochoric condition (5) is met, as expected, and it is clear that admissible generating functions $F=F_{2}(x, Y, t)$ must be such that the mixed partial derivative $\partial^{2} F / \partial x \partial Y$ does not vanish in the body.

We observe, from Eq. (29), that the function

$$
F=x Y
$$

generates the identity transformation and that a harmonic generating function, i.e., one for which

$$
\partial^{2} F / \partial x^{2}+\partial^{2} F / \partial Y^{2}=0
$$

gives rise to a symmetric deformation gradient tensor, which implies that the deformation is a pure stretch, or a pure stretch and a rigid rotation through $\pi$ radians. As an example, the generating function

$$
F=\frac{1}{2} \ln \left(x^{2}+Y^{2}\right)
$$

gives

$$
y=x /\left(x^{2}+Y^{2}\right), \quad X=Y /\left(x^{2}+Y^{2}\right),
$$

or

$$
x^{2}=Y(1-X Y) / X, \quad y^{2}=X(1-X Y) / Y .
$$

Equations (33) give

$$
x X=y Y \text { and } \quad x y+X Y=1,
$$

so that this deformation carries radial lines $Y=m X$ into radial lines $y=x / m$ and hyperbolae $X Y=c$ into hyperbolae $x y=1-c$ and leaves the line $Y=X$ and the hyperbola $X Y=1 / 2$ unchanged.

Generating functions for isochoric axisymmetric motions. Representations of the isochoric axisymmetric motions (9) in terms of generating functions are obtained directly from the previous results by observing that the system of Eqs. (6)-(10) is reduced to the system (1)-(5) by the coordinate transformations

$$
x=r^{2}, \quad y=z ; \quad X=R^{2}, \quad Y=Z .
$$

Thus we have, for example,

$$
z=\partial F / \partial r^{2}, \quad Z=-\partial F / \partial R^{2} ; \quad F=F_{1}\left(r^{2}, R^{2}, t\right)
$$

or

$$
r^{2}=-\partial F / \partial z, \quad R^{2}=\partial F / \partial Z ; \quad F=F_{4}(z, Z, t) .
$$

Discussion. The results obtained here substantiate the intuitive idea that it should be possible to represent an isochoric plane or axisymmetric finite motion, or static deformation, in terms of a single scalar function. The generating function involves one coordinate 
in each of two different configurations of the body. Thus, it may not be a very suitable representation for solving boundary value problems for specific materials. Even for a simple elastic response law, such as that for a neo-Hookean solid, calculation of the stress from Eq. (29), and substitution in the equation of equilibrium, leads to a rather complicated nonlinear partial differential equation for the function $F(x, Y)$.

\section{REFERENCE}

[1] H. Goldstein, Classical mechanics, Addison-Wesley, Reading, Mass., 1950 